\title{
Bacterial cell disruption by process of hydrodynamic cavitation. Mechanisms and application
}

\author{
Anna Nedbailo., Georgiy Ivanitsky. \\ Institute of Engineering Thermophysics of NAS of Ukraine, Ukraine, Kyiv, 2a, Zhelyabova Str., \\ anna.nedbailo18@gmail.com
}

\begin{abstract}
The article presents complete understanding of bacterial cell disruption process under hydrodynamical cavitation (HC) condition. Analysis of present literature has shown that the process of cavitation is widely used in the food, chemical, pharmaceutical, and biological industries and is effective from ecological and energy point of view. Novel studies presents mechanisms, which ascertain fact of complete or partially cell wall destruction, but not explain reasons of this. The article proposes mechanisms and their theoretical justification for the extraction and sterilization process, and the thermophysical parameters necessary for conducting a particular process are determined.
\end{abstract}

Hydrodynamic cavitation, disruption, cell wall, sterilization

\section{Introduction}

Many scientific papers are devoted to the use of hydrodynamic cavitation in sterilization technologies. Researchers of cavitation effects on biological cells describe the high efficiency of HC treatment. The advantages of such processing are the reduction of the processing time, reduction of energy consumption and the amount of chemical reagents, environmental friendliness of the technology. The results of existing microscopic studies indicate that under HC conditions, the pasteurization or sterilization effect is provided by the destruction of the cell wall of microorganisms, as a result of the impact and shear mechanisms at the final stage of compression of the cavitation cluster [1, 3]. However, the detailing of these mechanisms at the micro level and the quantitative calculation of the force effect of cavitation on the cells were not carried out. The clear understanding of $\mathrm{HC}$ process are necessary for investigation the effects of cavitation on biological cells. The works $[1,2]$ describe the thermophysical foundations of the cavitation process, show methods for calculating hydrodynamic and thermodynamic parameters that cause the partial or complete suppression of microflora.

\section{Analysis of the cell structure and the mechanisms of its destruction}

Cytoplasm is contained inside the cell, which is separated from the surrounding fluid by a thin $(8 \ldots 10 \mathrm{~nm}$ ) semi-permeable plasma membrane with a pore diameter (ion channels) of $0.4 \ldots 0.5 \mathrm{~nm}$. The osmotic pressure of the cytoplasm $(0.3 \ldots 1.0 \mathrm{MPa})$ acts on the membrane surface inside. The cell membrane is surrounded by a thin and durable cell wall that prevents tearing and stretching of the plasma membrane. The pore size of the cell wall is $4 \ldots 5 \mathrm{~nm}$, it is permeable to water, salts and low molecular weight compounds. The strength of the cell walls of microorganisms reaches $100 \mathrm{MPa}$, which is comparable to the tensile strength of some steel grades [4]. The role of cavitation mechanisms in the inactivation of bacterial cells is far from being studied. The death of microorganisms is associated with hydromechanical, thermal, chemical, electrical effects of cavitation or with the combined effect of these factors.

For analyzing the impact of shock pulses of pressure of a cavitation cluster we are assumed that the bacterial cell in the form of a sphere or a cylindrical rod is in equilibrium with a liquid medium with pressure $p_{l 0}=0,1 \mathrm{MPa}$. On the inner surface of the cell wall with thickness $h_{c}$ through the elastic semipermeable membrane adjacent to it osmotic pressure $\pi \gg P_{l 0}$ acts as a result, tensile stresses arise in the walls. Tangential magnitudes $\sigma_{t}$ and normal stress $\sigma_{n}$ in the bacterial bacillus cell wall is estimated using the formulas:

$$
\sigma_{t}=p_{c} R_{c} / h \text { and } \sigma_{n}=p_{c} R_{c} / 2 h,
$$

and the normal tension in the spherical bacterial wall is as follows:

$$
\sigma_{n}=p_{c} R_{c} / 2 h .
$$


where $R_{c}$ - the radius of the cylindrical part of the bacteria, and in (2) - radius of the sphere.

Parameter $p_{c}$ - pressure acting on the inner surface of the wall. For large values of the $p_{c}$ tensile stress may exceed the tensile strength of the wall, which leads to its destruction. According to these formulas, the calculation of the strength of the wall of such microorganisms S. aureus, L. lactis, Lactobacillus, E. coli was carried out, which was $1.20 \mathrm{MPa}, 2.67 \mathrm{MPa}, 1.75 \mathrm{MPa}$ and $0.8 \mathrm{MPa}$ respectively.

With a sharp increase in pressure in the surrounding fluid $\left(p_{l}>p_{l 0}\right)$, the internal pressure on the cell wall decreases sharply, which leads to a weakening of the stresses acting in it. When $p_{l}>\pi$ the magnitude of tensile stresses in the cell $\sigma \rightarrow 0$. The excess pressure applied to the outer surface of the plasma membrane leads to compression of the cytoplasm and an increase in the internal pressure in it from $\pi$ to $p_{l}=\pi+\Delta p$. As a result, the potential energy of the compressed cytoplasm increases, and after a sharp relief of external pressure $p_{l}$, it is realized in the form of a shock effect on the inner surface of the cell wall and cell death.

Studies of the effects of shear stresses [4] showed that the shear rate required for the destruction of gram-positive bacteria in the form of a sphere, lie in the range of $8 \cdot 10^{6} \ldots 9 \cdot 10^{7} \mathrm{~s}^{-1}$, and for the destruction of gram-positive bacteria in the form of a stick, a shear rate of about $5 \cdot 10^{3} \ldots 7 \cdot 10^{5}$ $\mathrm{s}^{-1}$. As is well known, cavitation processes are characterized by the appearance of radial currents caused by the growth and collapse of cavitation bubbles, which also has a dynamic effect on bacterial cells.

\section{Experimental}

To confirm the validity of these mechanisms, experimental studies have been carried out on the suppression of the microflora of milk on equipment that implements a combination of hydrodynamic effects (cavitation, explosive boiling up) and thermal effects. In this technology, cavitation effects are implemented in the impeller of centrifugal pumps, which are used as product pumps for pumping a liquid product, and according to the technology conditions they work nominally in cavitation mode. Milk is consistently subjected to a double cavitation effect. The first stage of cavitation treatment occurs in the pump, which operates in the temperature range of $50 \ldots 60{ }^{\circ} \mathrm{C}$. After leaving the pump, the milk undergoes heat treatment in a pasteurizer $\left(T_{l \max }=80 \ldots 95{ }^{\circ} \mathrm{C}\right)$. The second stage of cavitation treatment is carried out before leaving the apparatus in a centrifugal pump operating in the temperature range of $25 \ldots 30^{\circ} \mathrm{C}$. The level of cavitation in each of the pumps, as shown below, depends on the temperature mode of treatment.

\section{Results and discussion}

The total bacterial contamination decreases by more than two orders of magnitude after heating the milk from $4^{\circ} \mathrm{C}$ to $50 \ldots 60^{\circ} \mathrm{C}$ and cavitation treatment in the first pump. After heat treatment in a pasteurizer at $T_{l}=95^{\circ} \mathrm{C}$, the total bacterial contamination decreases even more than an order of magnitude. After passing through the zone of adiabatic boiling up and subsequent cavitation treatment in the second pump at $T_{l}=30^{\circ} \mathrm{C}$, seeding decreases by almost an order of magnitude. The number of bacteria S.aureus and E. coli fig. $1(\mathrm{a}, \mathrm{b})$ decreases to zero already at the first stages of processing, since they are characterized by low wall strength. There are practically no surviving cells of the gram-positive Lactobacillus in the samples taken at the exit from the apparatus, while their content in the samples taken after the pasteurizer is still quite high. Survived after processing cells L.lactis, with a stronger cell wall determine, mainly, the level of residual contamination of milk.

The obtained data indicate that a significant contribution to the inactivation of microorganisms makes cavitation created in centrifugal pumps. Most researchers are inclined to believe that the role of cavitation in the destruction of bacterial cells lies in the action of the shock and shear mechanisms at the final stage of compression of the cavitation cluster $[1,4]$.

Pressure pulses calculated according to the data of [5] calculated within the model (fig. 2), for different values of liquid temperature $T_{l}$, [1], emitted by a cavitation cluster in the pump impeller at 
the moment of bubble collapse. The amplitude of the pulses $p_{l \max } \max$ decreases from $2.3 \mathrm{MPa}$ to 1.2 $\mathrm{MPa}$ with increasing liquid temperature from 20 to $60^{\circ} \mathrm{C}$. The magnitude of the pressure pulses is insufficient to destroy the solid cell wall of bacteria L.lactis, which is consistent with the results of the experiment. At the same time, the obtained results show that the intensive suppression of the vital activity of Lactobacillus bacteria in the final stage of treatment at $T_{l}=30^{\circ} \mathrm{C}$ observed in the experiment can be explained by the action of cavitation in the second pump when the condition $p_{l \max }=p_{c r}$ is fulfilled.

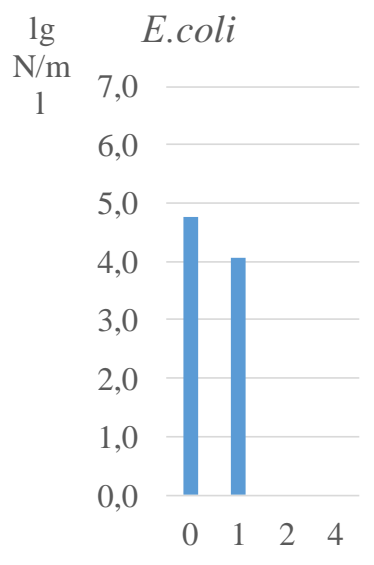

a)

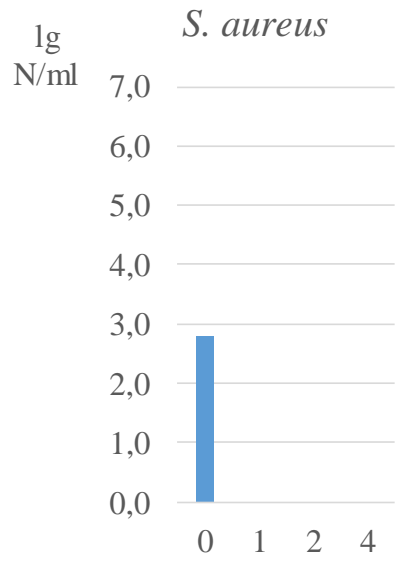

b)

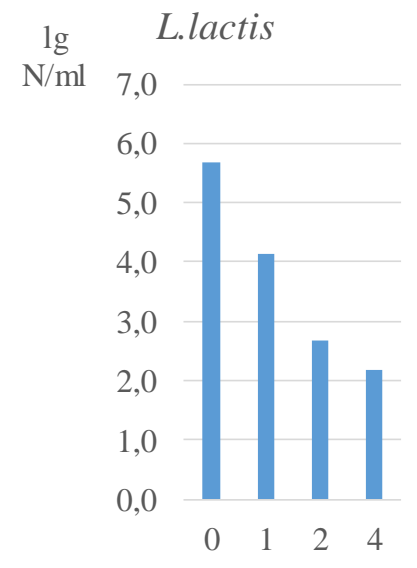

c)

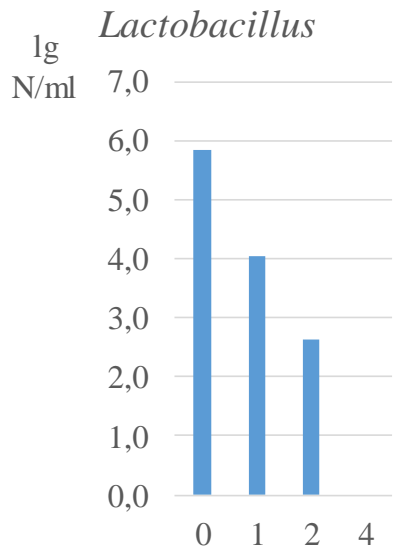

d)

Fig. 1. Amount of bacterial cells at the stages of thermo-hydrodynamic treatment: 0 - initial sample; 1 - first stage of the cavitation influence, 2 - thermal treatment; 3 - second stage of the cavitation influence.

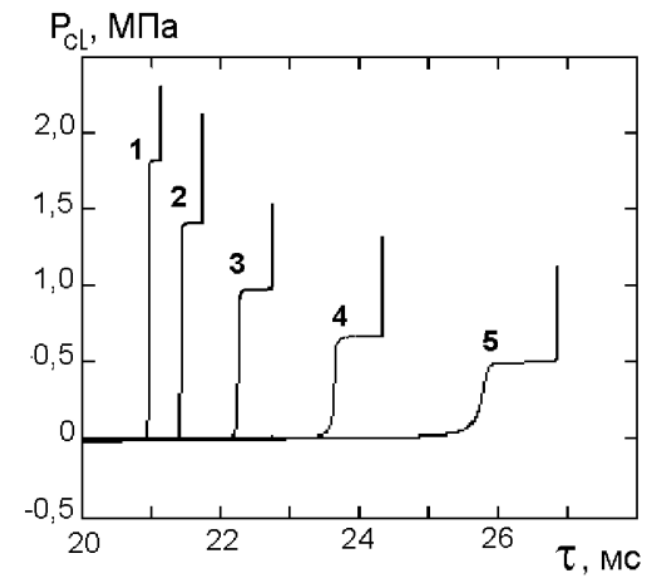

Fig. 2. The dependence of pressure in time in the volume of the cavitation cluster at different values of the temperature of the liquid $T_{l}: 1-20^{\circ} \mathrm{C} ; 2-30^{\circ} \mathrm{C} ; 3-40^{\circ} \mathrm{C} ; 4-50^{\circ} \mathrm{C} ; 5-60^{\circ} \mathrm{C}$. The pulses correspond to the moment of bubble collapse.

The system of equations of the model of the dynamics of an ensemble of vapor bubbles is presented at the work [1], which makes it possible to calculate instantaneous values of pressure and velocity vector at local points inside the $3 \mathrm{D}$ cavitation cluster at all stages of its evolution. Using the model, one can estimate the level of shear stresses under various conditions of cluster formation and development. In fig. 3, a presents the calculated data on the change in the magnitude of the velocity in the XY plane passing through the center of the cluster in the X direction over a section of $0.3 \mathrm{~mm}$ in length for eight consecutive values of the $\mathrm{Y}$ coordinate with an interval of $10 \mu \mathrm{m}$. In fig. $3 \mathrm{~b}$ for the same time point shows the change in the direction of the velocity vector at the same local points.

The presented data indicate a strong vorticity of microcurrents within the cluster. Calculations show that shear rates at local points of the cluster at the boundaries of the interaction of microflows 
are $10^{5} \ldots 10^{6} \mathrm{~s}^{-1}$, which is comparable with the values of this parameter achieved in rotary apparatuses and in valve homogenizers. With regard to low-viscosity fluids (water, milk), this corresponds to shear stresses of the order of $0,001 \ldots 0,1 \mathrm{MPa}$.

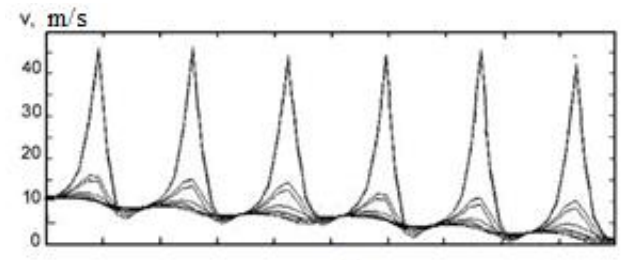

a)

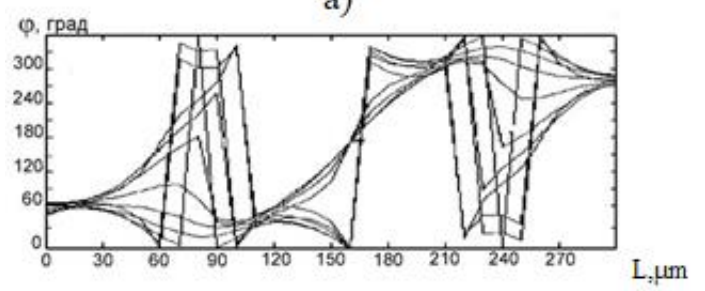

b)

Fig. 3. a) - the change in the magnitude of the velocity vector along the $X$ direction in the 3D cavitation cluster for eight consecutive values of the Y coordinate with an interval of $10 \mu \mathrm{m}$; b)changing the direction of the velocity vector in the same $\mathrm{X}$ direction for the same $\mathrm{Y}$ coordinate values.

According to many researchers, shear stresses of this level can destroy the cell walls of even gram-positive bacteria [6]. The results we obtained are consistent with the results of the research of other authors. In [4], it is noted that the values of shear rates necessary for the destruction of cells of gram-positive bacteria in the form of a sphere are in the range of $3 \cdot 10^{6} \ldots 9 \cdot 10^{7} \mathrm{~s}^{-1}$, and for the destruction of gram-positive bacteria in the form of a rod - in the range of $2 \cdot 10^{4} \ldots 7 \cdot 10^{6} \mathrm{~s}^{-1}$. For the destruction of gram-negative rods, shear velocities of the order of $5 \cdot 10^{3} \ldots 7 \cdot 10^{5} \mathrm{~s}^{-1}$ are sufficient.

\section{Conclusion}

This study shows that technologies based on the methods of $\mathrm{HC}$ can be applied to bacterial sononation of various media. The degree of suppression of microflora depends on the parameters of cavitation, which must be individually selected depending on the purpose of treatment. Low process temperature allows processing thermolabile raw materials. The significant contribution of cavitation to the suppression of undesirable microflora of milk allows to reduce the pasteurization temperature. The reduction of excessive thermal effects on the thermolabile product contributes to its quality indicators, as well as the energy efficiency of its processing.

\section{References}

[1] Dolinsky A.A., Ivanitsky G.K. Heat and mass transfer and hydrodynamics in vapor-liquid dispersed media. Kiev, Naukova dumka, 2008.

[2] Jitendra Carpenter, Mandar Badve, Sunil Rajoriya, Suja George, Virendra Kumar Saharan and Aniruddha B. Pandit.. Hydrodynamic cavitation: an emerging technology for the intensification of various chemical and physical processes in a chemical process industry. Reviews in Chemical Engineering. Vol. 10. pp. 3 - 37, 2016.

[3] Farid Chemat, Natacha Rombaut, Anne-Gaëlle Sicaire, Alice Meullemiestre, Anne-Sylvie Fabiano-Tixier, Maryline Abert-Vian. Ultrasound assisted extraction of food and natural products. Mechanisms, techniques, combinations, protocols and applications. A review. Ultrasonics Sonochemistry. Vol. 34. pp.540-560, 2017.

[4] Chisti Yu., Moo-young M. Disruption of microbial cells for intracellular products. Review Enzyme Microbiol. Techn. Vol. 8., pp.194 - 204, 1986.

[5] Ivanitsky G.K., Nedbaylo A.E. Analytical study of cavitation in the impeller of centrifugal pumps. Industrial Heat Engineering, Kiev, vol. 34(2), pp.40 - 47, 2011.

[6] Ashokkumar M., Krasulya O., Rink R. A New Look at Cavitation and the Applications of Its Liquid-Phase Effects in the Processing of Food and Fuel, Appl. Phys. Research, vol. 4(1), pp. $19-29,2012$. 